Case Report

\title{
Disseminated Histoplasmosis and Secondary Hemophagocytic Syndrome in a Non-HIV Patient
}

\author{
Muhammad Kashif, ${ }^{1}$ Hassan Tariq, ${ }^{1}$ Mohsin Ijaz, ${ }^{2}$ and Jose Gomez-Marquez ${ }^{2}$ \\ ${ }^{1}$ Department of Medicine, Bronx Lebanon Hospital Center, 1650 Selwyn Avenue, Suite No. 10 C, Bronx, NY 10457, USA \\ ${ }^{2}$ Division of Pulmonary and Critical Care Medicine, Department of Medicine, Bronx Lebanon Hospital Center, 1650 Selwyn Avenue, \\ Suite No. 12 F, Bronx, NY 10457, USA
}

Correspondence should be addressed to Muhammad Kashif; mkashif@bronxleb.org

Received 28 May 2015; Revised 17 July 2015; Accepted 26 July 2015

Academic Editor: Mabrouk Bahloul

Copyright (C) 2015 Muhammad Kashif et al. This is an open access article distributed under the Creative Commons Attribution License, which permits unrestricted use, distribution, and reproduction in any medium, provided the original work is properly cited.

Histoplasma duboisii, a variant of Histoplasma capsulatum that causes "African histoplasmosis," can be resistant to itraconazole, requiring intravenous amphotericin B treatment. Rarely, these patients do not respond to intravenous antifungal therapy, and in such cases, patients may progress to develop secondary hemophagocytic lymphohistiocytosis (HLH). We present a case of a 34-yearold male patient with sickle cell disease who presented with a 5-month history of an enlarging painless axillary mass, persistent low grade fevers, night sweats, weight loss, and anorexia. An excisional biopsy of the right axillary lymph node revealed yeast and granulomas consistent with histoplasma infection. He was started on oral itraconazole. After 4 weeks of therapy, laboratory evaluation revealed worsening anemia, thrombocytopenia, and transaminitis. Due to failure of oral therapy, he was admitted for intravenous amphotericin B treatment. During his hospital course anemia, thrombocytopenia, and transaminitis all worsened. A bone marrow biopsy was done that was consistent with HLH. His clinical status continued to deteriorate, developing multiorgan failure and disseminated intravascular coagulation. He unfortunately had a cardiorespiratory arrest after eight days of admission and passed away.

\section{Introduction}

Disseminated histoplasmosis $(\mathrm{DH})$ is a chronic granulomatous disease caused by Histoplasma capsulatum, usually in immunocompromised patients. It typically responds to treatment with oral antifungal therapy $[1,2]$. However, Histoplasma duboisii, a variant of Histoplasma capsulatum that causes "African histoplasmosis," is endemic to tropical and temperate areas of sub-Saharan Western Africa and Madagascar and can be resistant to itraconazole, requiring intravenous amphotericin B treatment [3]. Rarely, these patients do not respond to intravenous antifungal therapy and in such cases, patients may progress to develop secondary hemophagocytic lymphohistiocytosis (HLH) $[4,5]$. This diagnosis should be suspected in patients with persistent fever, splenomegaly, cytopenia, hypertriglyceridemia, hypofibrinogenemia, and elevated ferritin level. HLH is associated with a high mortality; hence, early recognition and initiation of treatment is crucial.

\section{Case Presentation}

A 34-year-old male presented to our outpatient clinic with painless, progressively enlarging right axillary lump that he noticed 5 months prior to presentation. His medical history was significant for sickle cell disease, and his last hospitalization had been 7 years ago for a sickle cell pain crisis. His symptoms included persistent low grade fevers, night sweats, weight loss, and anorexia. He did not have cough, dyspnea, arthritis, chest pain, or abdominal pain. The patient had no sick contacts and no contacts with animals. He had immigrated to United States from Nigeria one year prior to his presentation and had a negative tuberculin skin test. He had never smoked tobacco and had no toxic habits. He had no reported allergies. His medications included folate, iron, and multivitamin supplements.

Physical examination revealed an emaciated man. Vitals showed a fever $100.2 \mathrm{~F}$, pulse rate $88 / \mathrm{min}$, respiratory rate 


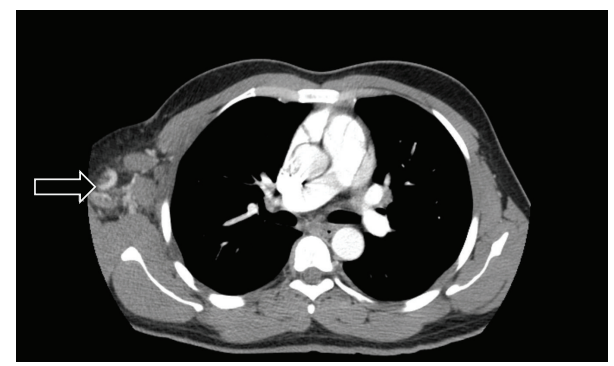

FIgURE 1: Computed tomography showing extensive right axillary lymphadenopathy (arrow).

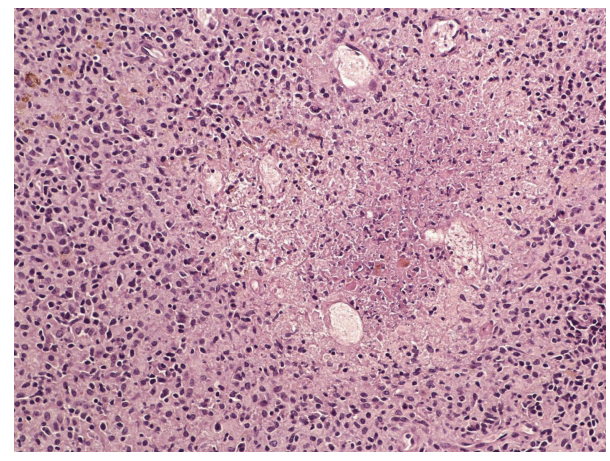

FIGURE 2: Lymph node with necrotizing granuloma comprised of necrotic center surrounded by epithelioid cells and lymphocytes.

$18 / \mathrm{min}$, and blood pressure $120 / 80 \mathrm{~mm}$ of hg. He was saturating $95 \%$ on ambient air. He had conjunctival pallor and palpable nontender right axillary lymphadenopathy.

Abdominal exam revealed hepatosplenomegaly. There was bilateral air entry on auscultation of lungs with no adventitious sounds. Precordial examination revealed normal heart sounds with no murmur, rub, or gallop.

Computerized tomography (CT) of chest with contrast revealed multiple lung nodules, a large axillary lymph node (Figure 1), and small multiple liver masses. An excisional biopsy of the right axillary lymph node revealed yeast and granulomas consistent with histoplasma infection (Figures 2 and 3). He was started on oral itraconazole. Laboratory evaluation after 4 weeks of treatment revealed worsening anemia, thrombocytopenia, and transaminitis. Due to the failure of oral itraconazole treatment as outpatient, he was admitted for intravenous amphotericin B treatment.

His labs on initial presentation and admission and during hospitalization are summarized in Table 1. Other pertinent laboratory results are shown in Table 2.

The patient was started on liposomal amphotericin B. Hematology was consulted for worsening anemia and thrombocytopenia, and a bone marrow biopsy was performed. His clinical status started to deteriorate on day five of admission, requiring ICU transfer for sepsis with high grade fever, tachycardia, drowsiness, and unilateral alveolar infiltrates on chest radiography. On day six, he developed septic shock and was started on vasopressors. The bone marrow biopsy revealed

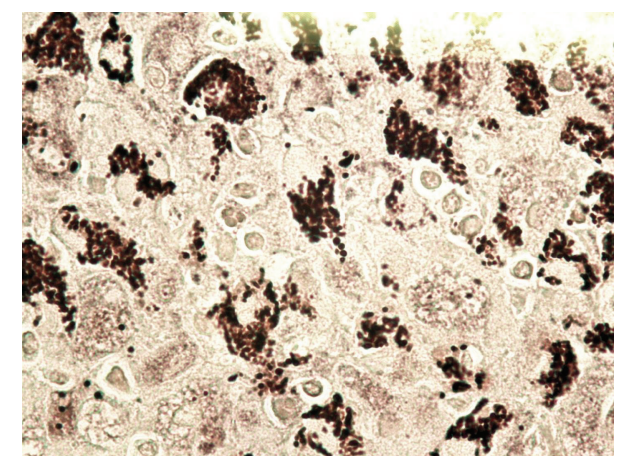

FIGURE 3: Silver stain on lymph node showing large histiocytes containing abundant yeasts (Histoplasma).

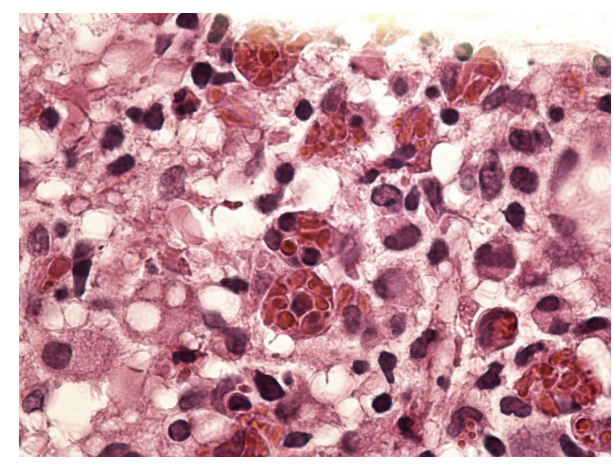

FIGURE 4: Hemophagocytic lymphohistiocytosis: bone marrow showing lymphohistiocytic infiltrate with extensive hemophagocytosis.

a lymphohistiocytic infiltrate with extensive hemophagocytosis consistent with hemophagocytic syndrome (Figure 4 ). The patient was started on a standard treatment protocol for HLH consisting of dexamethasone and etoposide. Unfortunately, his clinical status continued to worsen, developing multiorgan failure and disseminated intravascular coagulation. He had a cardiopulmonary arrest on day eight and passed away.

\section{Discussion}

$\mathrm{DH}$ is a chronic granulomatous disease caused by the dimorphic fungus Histoplasma capsulatum (HC). The fungus is ubiquitous and lives particularly in soil that contains large amounts of bird or bat droppings. It is endemic in the Central and Eastern United States, Central and South America, Africa, Asia, and Australia. It enters the human body through spore inhalation. Immunocompetent patients usually develop a self-limiting disease, manifested by fever, cough, and fatigue [1]. In patients with defective immune systems, the infection can become severe and spread hematogenously to other organs. Disseminated disease manifests as high grade fever, fatigue, weight loss, hepatosplenomegaly, and lymphadenopathy, mimicking the features of tuberculosis [2]. Skin lesions, pancytopenia, and anemia are more common among the immunocompromised. The disease is 
TABLE 1: Laboratory values on initial presentation and admission and during hospitalization.

\begin{tabular}{|c|c|c|c|c|}
\hline Labs & $\begin{array}{c}\text { Initial } \\
\text { presentation }\end{array}$ & $\begin{array}{c}\text { On } \\
\text { admission } \\
\text { Day } 1 \\
\end{array}$ & Days 3-4 & Day 8 \\
\hline Hemoglobin (g/dL) & 12.3 & 8.9 & 6.9 & 4.9 \\
\hline Hematocrit (\%) & 38.2 & 26.6 & 22 & 13.7 \\
\hline WBC count $(\mathrm{k} / \mathrm{uL})$ & 5.6 & 4.9 & 7.9 & 10 \\
\hline Platelet (k/uL) & 283 & 144 & 92 & 48 \\
\hline Reticulocyte \% & & 3.7 & 3.6 & \\
\hline $\begin{array}{l}\text { D dimer assay } \\
(\mathrm{ng} / \mathrm{mL})\end{array}$ & & & 4162 & 26206 \\
\hline $\begin{array}{l}\text { Fibrinogen } \\
(\mathrm{mg} / \mathrm{dL})\end{array}$ & & & 254 & 69 \\
\hline INR & 1.2 & 1.3 & 2.1 & 4.9 \\
\hline $\begin{array}{l}\text { Activated partial } \\
\text { thromboplastin } \\
\text { time (seconds) }\end{array}$ & 31 & 31.3 & 34 & 59 \\
\hline $\mathrm{LDH}$ & 632 & 777 & 1466 & \\
\hline Haptoglobin & & 2 & 1 & \\
\hline$(\mathrm{mL} / \mathrm{min})$ & 87 & 121 & 87 & 20 \\
\hline Sodium (mEq/L) & 138 & 136 & 145 & 142 \\
\hline Potassium (mEq/L) & 4 & 4.4 & 4.2 & 4.4 \\
\hline Chloride (mEq/L) & 100 & 97 & 112 & 106 \\
\hline $\begin{array}{l}\text { Bicarbonate } \\
(\mathrm{mEq} / \mathrm{L})\end{array}$ & 30 & 29 & 17 & 6 \\
\hline Calcium (mg/dL) & 10 & 8.6 & 8.2 & 7.7 \\
\hline $\begin{array}{l}\text { Phosphorus } \\
\text { (mg/dL) }\end{array}$ & & 4 & 4.2 & 8.5 \\
\hline Glucose (mg/dL) & 80 & 101 & 110 & 121 \\
\hline BUN (mg/dL) & 14 & 29 & 21 & 36 \\
\hline Creatinine (mg/dL) & 0.8 & 0.9 & 0.8 & 3 \\
\hline ALT (unit/L) & 70 & 184 & 3050 & 5058 \\
\hline AST (unit/L) & 59 & 214 & 6171 & 16637 \\
\hline $\begin{array}{l}\text { Alkaline } \\
\text { phosphatase } \\
\text { (unit/L) }\end{array}$ & 95 & 139 & 329 & 763 \\
\hline $\begin{array}{l}\text { Total protein } \\
(\mathrm{g} / \mathrm{dL})\end{array}$ & 7.7 & 5.5 & 6.4 & 3.4 \\
\hline Albumin (g/dL) & 4.8 & 3.4 & & 1.8 \\
\hline $\begin{array}{l}\text { Total bilirubin } \\
(\mathrm{mg} / \mathrm{dL})\end{array}$ & 0.7 & 1.2 & 6.3 & 8.1 \\
\hline $\begin{array}{l}\text { Direct bilirubin } \\
(\mathrm{mg} / \mathrm{dL})\end{array}$ & 0.3 & 0.6 & 5.1 & 3.1 \\
\hline $\begin{array}{l}\text { Lactic acid } \\
(\text { mmoles/L) }\end{array}$ & & 3 & 9.6 & 18 \\
\hline $\begin{array}{l}\text { Ammonia } \\
\text { (umole/L) }\end{array}$ & & & 126 & \\
\hline Ferritin (ng/mL) & & & 7493 & \\
\hline $\begin{array}{l}\text { Triglycerides } \\
(\mathrm{mg} / \mathrm{dL})\end{array}$ & 133 & 145 & 135 & \\
\hline
\end{tabular}

milder in the immunocompetent hosts with a higher cure rate [6].
TABLE 2: Laboratory work-up results.

\begin{tabular}{lc}
\hline Test & Result \\
\hline CD4 + T help cells/UL & $496(53 \%)$ \\
HIV serology & Negative \\
CMV IgM & Negative \\
[HTLV-I] and [HTLV-II] & Nonreactive \\
EBV VCA IgM & $<0.91$ \\
Serum histoplasma antigen & Negative \\
Urine histoplasma antigen & Negative \\
AFB blood culture & Negative \\
AFB tissue cultures & Negative \\
Blood cultures and urine cultures & Negative \\
Blastomyces serology & Negative \\
Coccidioides serology & Negative \\
Parvovirus serology & Negative \\
Echinococcus serology & Negative \\
Tissue viral culture & Negative \\
BAL culture & Negative \\
Hepatitis B surface antigen & Negative \\
Hepatitis B surface antibody & Positive \\
Hepatitis C & Negative \\
Serum Ceruloplasmin (mg/dL) & 59 \\
Anti-HEV IgM & Negative \\
Urine Legionella antigen & Negative \\
Cryptococcal antigen & Negative \\
Immunoglobulin G level (mg/dL) & 797 \\
\hline
\end{tabular}

Antigen detection appears to be the most sensitive rapid assay, which detects HC in $86-90 \%$ of AIDS patients [7]. The sensitivity of the urine histoplasma antigen assay in the immunocompetent patient is not well established, although it is reported to be as high as $80 \%$ compared with $82 \%$ in the immunosuppressed non-AIDS patient [8]. Polymerase chain reaction assays, blood cultures, and direct microscopic examination of specimens such as bronchial aspirates, bone marrow biopsy, or peripheral blood smear are also utilized [9], with a positive culture being the gold standard for diagnosing $\mathrm{DH}$. In this case the urine and serum histoplasma antigen were negative, requiring histopathology for diagnosis.

Antifungal agents effective for treatment of histoplasmosis include amphotericin $\mathrm{B}$ formulations and itraconazole. Amphotericin B formulations are used for patients who have severe pulmonary or disseminated forms of histoplasmosis. Amphotericin B is generally used initially until the patient has shown a favorable response and can take an oral antifungal agent; then itraconazole is given for the remainder of the treatment course. Itraconazole given orally is preferred for patients who have mild-to-moderate histoplasmosis and as a step-down therapy after the initial response to amphotericin B. Depending on the severity of the infection and the immune status, the course of treatment can range from 3 months to 1 
year. Response rate for primary therapy with itraconazole in early studies was $100 \%$ for disseminated histoplasmosis [10].

Histoplasma capsulatum var. duboisii, which causes African histoplasmosis, is endemic to tropical and temperate areas of sub-Saharan Western Africa and Madagascar and is often resistant to oral therapy with itraconazole [3]. Our patient had migrated from Nigeria, and considering the treatment failure with itraconazole, our suspicion is that he may have had the duboisii variant of histoplasmosis. From the onset, treatment with liposomal amphotericin B is recommended in these patients, with favorable clinical outcomes [3].

Hemophagocytic syndrome refers to a wide array of related diseases and can be primary or secondary [11]. Primary HLH syndrome involves a genetic defect caused by mutation of the perforin gene. It includes two categories: familial hemophagocytic lymphohistiocytosis and immune deficiency syndrome, which conversely also includes ChédiakHigashi syndrome, Griscelli syndrome, X-linked lymphoproliferative syndrome type 1, Wiskott-Aldrich syndrome, severe combined immunodeficiency, and Hermansky-Pudlak syndrome. Secondary HLH is associated with a number of conditions including viral infections (29\%), other types of infections (20\%), malignancies (27\%), rheumatologic disorders (7\%), and immune deficiency syndromes (6\%) [12]. HLH can occur in all age groups without predilection for race or sex [13].

HLH is believed to be the result of unrestrained macrophage activity. Macrophages serve as antigen presenting cells to lymphocytes for either direct destruction of antigens or antibody development. In the various forms of HLH, macrophages become activated and secrete cytokines. Cytokines, in turn, can cause organ damage when excreted in excessive amounts, resulting in systemic inflammatory response, immune dysregulation, and tissue damage. In addition, chronic antigen stimulation in the setting of viral infections leading to cytotoxic T-cell dysfunction has also been postulated to drive HLH. Natural killer- (NK-) cells function by directly destroying damaged or infected cells, independent of the major histocompatibility complex (MHC). Cytotoxic Tlymphocytes, while being similar to NK-cells, kill autologous cells carrying the foreign antigens associated with MHC Class I. Defects in NK-cell function may vary within the various types of HLH. Genetic forms of HLHs are due to defects in transport, processing, and function of cytotoxic granules in NK-cells and cytotoxic T-lymphocytes [14]. The ineffective antigen removal results in ongoing immune stimulation and inappropriate hemophagocytosis. The diagnosis of $\mathrm{HLH}$ requires fulfillment of one or both of the following criteria $[11,15]$ :

(1) a molecular diagnosis consistent with HLH;

(2) five of these eight findings:

(i) fever $\geq 38.5^{\circ} \mathrm{C}$,

(ii) splenomegaly,

(iii) peripheral blood cytopenia, with at least two of the following: hemoglobin $<9 \mathrm{~g} / \mathrm{dL}$ (for infants $<4$ weeks, hemoglobin $<10 \mathrm{~g} / \mathrm{dL}$ ), platelets
$<100,000 / \mathrm{microL}$, and absolute neutrophil count $<1000 / \mathrm{microL}$;

(iv) fasting triglycerides $>265 \mathrm{mg} / \mathrm{dL}$ and/or fibrinogen $<150 \mathrm{mg} / \mathrm{dL}$;

(v) hemophagocytosis in bone marrow, spleen, lymph node, or liver;

(vi) low or absent NK-cell activity;

(vii) ferritin $>500 \mathrm{ng} / \mathrm{mL}$;

(viii) elevated soluble CD25 (soluble IL-2 receptor alpha) two standard deviations above ageadjusted laboratory-specific norms.

Serum ferritin level is readily available test; with level above $10,000 \mu \mathrm{g} / \mathrm{L}$ has a sensitivity of $90 \%$ and specificity of $96 \%$ for the diagnosis of HLH. Flow cytometry can predict the presence of a genetic defect in most cases. When available, comparison of assays measuring IFN- $\gamma$, IL-10, and IL- 6 may be useful for distinguishing between bacterial sepsis, viral infections, and HLH in febrile patients [16]. Increased levels of serum ferritin, $\mathrm{LDH}$, and liver enzymes are also valuable parameters indicating HLH [11].

There have been 18 reported cases of disseminated histoplasmosis with the hemophagocytic syndrome [4]. Twelve of them occurred in HIV-infected patients and another case involved the development of HLH associated with a vasoocclusive crisis in a patient with hemoglobin $\mathrm{Sb}+$ thalassemia [17]. Infection-associated hemophagocytic syndrome can have a poor outcome. Predictors of a poor outcome include age older than 30 years, the presence of disseminated intravascular coagulation, elevated ferritin level, and anemia with accompanying thrombocytopenia [5].

Management of HLH presents many pitfalls: the condition is rare, it is not considered at onset, recognition can be difficult, and the clinical picture may be misleading [18]. Bone marrow aspiration should be considered early in the evaluation, with patients having pancytopenia, elevated ferritin levels, hypertriglyceridemia, and fever [19]. Early recognition is crucial for reasonable attempt at curative therapy to be made, given that treating an identified infection alone is not enough [15]. Life-threatening hyperinflammation, caused by excessive levels of cytokines, can be treated by corticosteroids which are cytotoxic for lymphocytes and inhibit the expression of cytokines and differentiation of dendritic cells. Most cases of infection-related HLH should be treated aggressively with standard HLH protocols that include dexamethasone, etoposide, and cyclosporine. However, delay in identification and treatment leads to fatal outcomes, as seen in this case. Duration of treatment is 8 weeks in patients with HLH who do not have an identifiable genetic defect [20]. Supportive care with prophylactic antibiotics, blood and platelet transfusions, fluid administration, and electrolyte repletion are all important steps in the treatment of HLH.

\section{Conclusions}

$\mathrm{DH}$ is more common in immunocompromised populations and can be complicated by atypical presentation such as HLH. A travel history to endemic areas is helpful in management. 
In the presence of pancytopenia, lymph node and bone marrow biopsies should be utilized early in the course of the evaluation to make the diagnosis of DH and HLH. Treatment involves a combination of antifungal and immunosuppressive therapy. Given the diagnostic difficulties and mortality associated with HLH, there should be a high index of suspicion in cases of disseminated histoplasmosis in sickle cell patients developing pancytopenia and not responding to standard treatment. Patients with infections should be monitored regularly for response since any uncontrolled infection can lead to HLH. A delay in the correct diagnosis of this complication can lead to an adverse outcome.

\section{Abbreviations}

ICU: Intensive care unit

CNS: Central nervous system

DH: Disseminated histoplasmosis

HLH: Hemophagocytic lymphohistiocytosis

AIDS: Acquired immunodeficiency syndrome

NK-cells: Natural killer cells.

\section{Conflict of Interests}

The authors of the paper declare that there is no conflict of interests regarding the publication of this paper.

\section{Authors' Contribution}

Muhammad Kashif and Hassan Tariq searched the literature and wrote the paper. Mohsin Ijaz conceived and edited the paper. Jose Gomez-Marquez supervised the patient treatment and critically revised and edited the paper. All authors have made significant contributions to the paper and have reviewed it before submission. All authors have confirmed that the paper is not under consideration for review at any other Journal. All authors have read and approved the final paper.

\section{References}

[1] C. A. Kauffman, "Pulmonary histoplasmosis," Current Infectious Disease Reports, vol. 3, no. 3, pp. 279-285, 2001.

[2] O. Seriki, W. I. Aderele, A. Johnson, and J. A. Smith, "Disseminated histoplasmosis due to Histoplasma capsulatum in two Nigerian children," Journal of Tropical Medicine and Hygiene, vol. 78, no. 12, pp. 248-255, 1975.

[3] A. Garcia-Guiñon, J. M. Torres-Rodríguez, D. T. Ndidongarte, F. Cortadellas, and L. Labrín, "Disseminated histoplasmosis by Histoplasma capsulatum var. duboisii in a paediatric patient from the Chad Republic, Africa," European Journal of Clinical Microbiology and Infectious Diseases, vol. 28, no. 6, pp. 697-699, 2009.

[4] P. R. Koduri, V. Chundi, P. DeMarais, B. A. Mizock, A. R. Patel, and R. A. Weinstein, "Reactive hemophagocytic syndrome: a new presentation of disseminated histoplasmosis in patients with AIDS," Clinical Infectious Diseases, vol. 21, no. 6, pp. 14631465, 1995.
[5] A. Sanchez, A. K. Celaya, and A. Victorio, "Histoplasmosisassociated hemophagocytic syndrome: a case report," AIDS Reader, vol. 17, no. 10, pp. 496-499, 2007.

[6] D. Deodhar, F. Frenzen, P. Rupali et al., "Disseminated histoplasmosis: a comparative study of the clinical features and outcome among immunocompromised and immunocompetent patients," National Medical Journal of India, vol. 26, no. 4, pp. 214-215, 2013.

[7] C. A. Kauffman, "Diagnosis of histoplasmosis in immunosuppressed patients," Current Opinion in Infectious Diseases, vol. 21, no. 4, pp. 421-425, 2008.

[8] L. J. Wheat, T. Garringer, E. Brizendine, and P. Connolly, "Diagnosis of histoplasmosis by antigen detection based upon experience at the histoplasmosis reference laboratory," Diagnostic Microbiology \& Infectious Disease, vol. 43, no. 1, pp. 29-37, 2002.

[9] C. Andre, J. Badoual, G. Kalifa, and J. Dubousset, "African histoplasmosis. A case," Archives françaises de Pédiatrie, vol. 41, no. 6, pp. 429-431, 1984.

[10] L. J. Wheat, A. G. Freifeld, M. B. Kleiman et al., "Clinical practice guidelines for the management of patients with histoplasmosis: 2007 update by the Infectious Diseases Society of America," Clinical Infectious Diseases, vol. 45, no. 7, pp. 807-825, 2007.

[11] I. Malinowska, M. Machaczka, K. Popko, A. Siwicka, M. Salamonowicz, and B. Nasiłowska-Adamska, "Hemophagocytic syndrome in children and adults," Archivum Immunologiae et Therapia Experimentalis, vol. 62, no. 5, pp. 385-394, 2014.

[12] M. R. George, "Hemophagocytic lymphohistiocytosis: review of etiologies and management," Journal of Blood Medicine, vol. 5, pp. 69-86, 2014.

[13] L. Zhang, J. Zhou, and L. Soko, "Hereditary and acquired hemophagocytic lymphohistiocytosis," Cancer Control, vol. 21, no. 4, pp. 301-312, 2014.

[14] C. Larroche and L. Mouthon, "Pathogenesis of hemophagocytic syndrome (HPS)," Autoimmunity Reviews, vol. 3, no. 2, pp. 6975, 2004.

[15] Z. Tothova and N. Berliner, "Hemophagocytic syndrome and critical illness: new insights into diagnosis and management," Journal of Intensive Care Medicine, 2014.

[16] X.-J. Xu, Y.-M. Tang, H. Song et al., "Diagnostic accuracy of a specific cytokine pattern in hemophagocytic lymphohistiocytosis in children," Journal of Pediatrics, vol. 160, no. 6, pp. 984.el990.e1, 2012.

[17] E. Kio, A. Onitilo, J. Lazarchick, M. Hanna, C. Brunson, and U. Chaudhary, "Sickle cell crisis associated with hemophagocytic lymphohistiocytosis," American Journal of Hematology, vol. 77, no. 3, pp. 229-232, 2004.

[18] S. Rajagopala, U. Dutta, K. S. P. Chandra, P. Bhatia, N. Varma, and R. Kochhar, "Visceral leishmaniasis associated hemophagocytic lymphohistiocytosis-case report and systematic review," Journal of Infection, vol. 56, no. 5, pp. 381-388, 2008.

[19] H. M. Madrigal-Jiménez and G. Hernández-Rivera, "Usefulness of bone marrow microscopic examination in HIV-infected patients with pancytopenia," Gaceta Médica de México, vol. 142, no. 1, pp. 13-17, 2006.

[20] J.-I. Henter, M. Arico, R. M. Egeler et al., "HLH-94: a treatment protocol for hemophagocytic lymphohistiocytosis. HLH study Group of the Histiocyte Society," Medical and Pediatric Oncology, vol. 28, no. 5, pp. 342-347, 1997. 


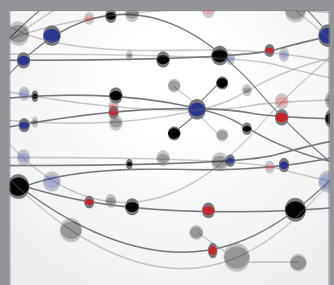

The Scientific World Journal
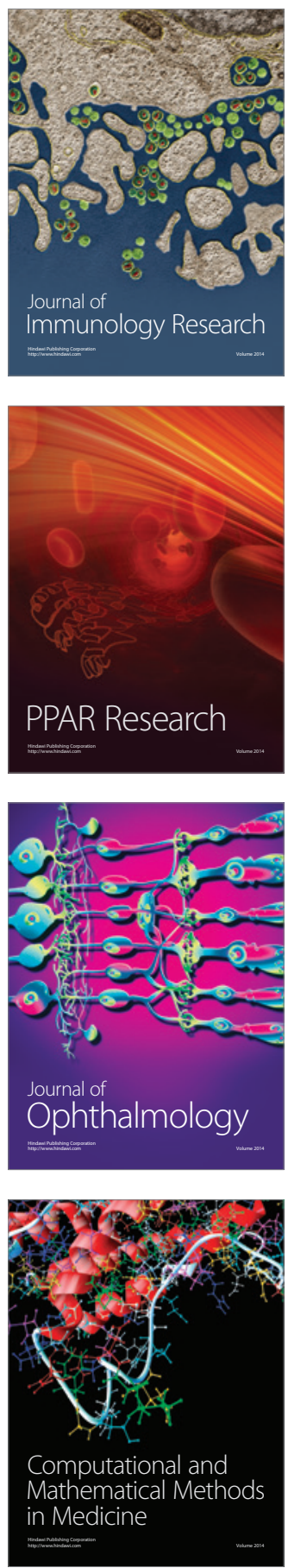

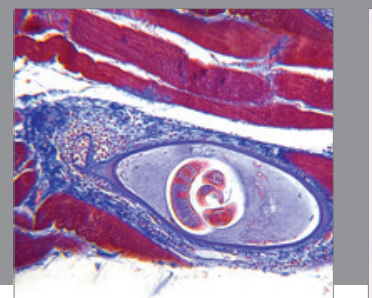

Gastroenterology

Research and Practice
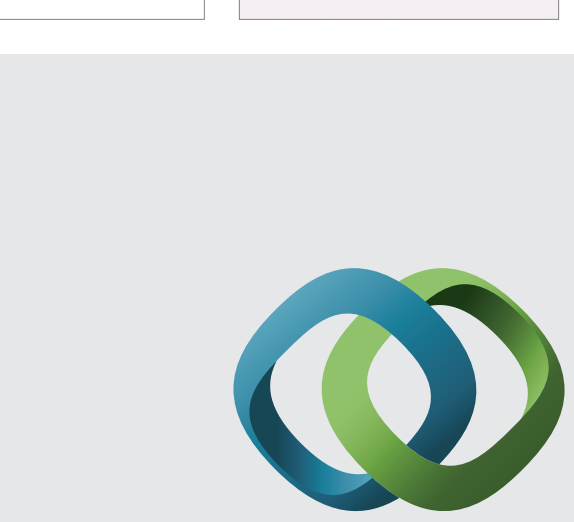

\section{Hindawi}

Submit your manuscripts at

http://www.hindawi.com
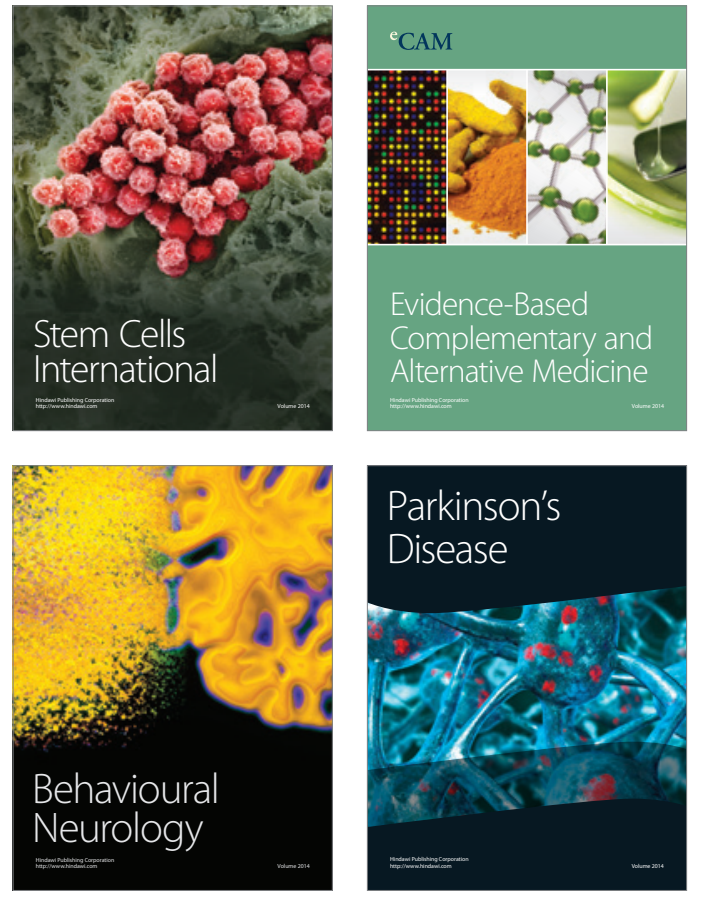
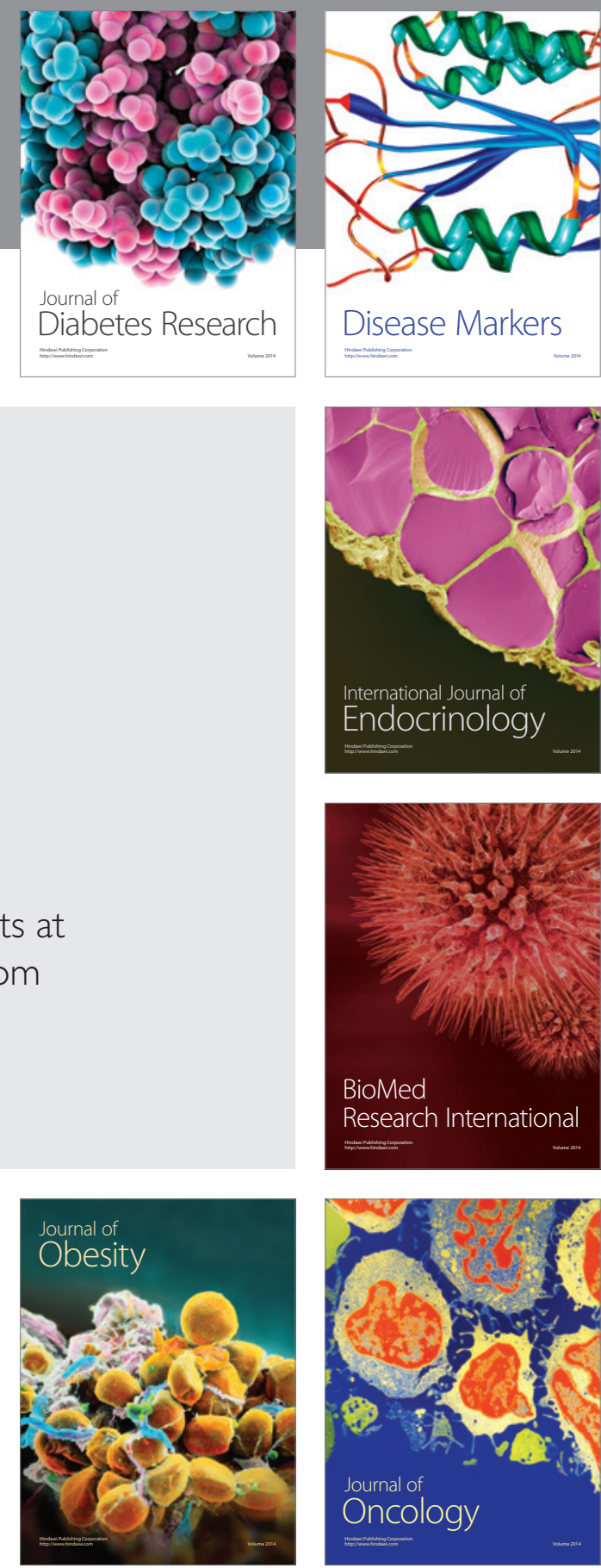

Disease Markers
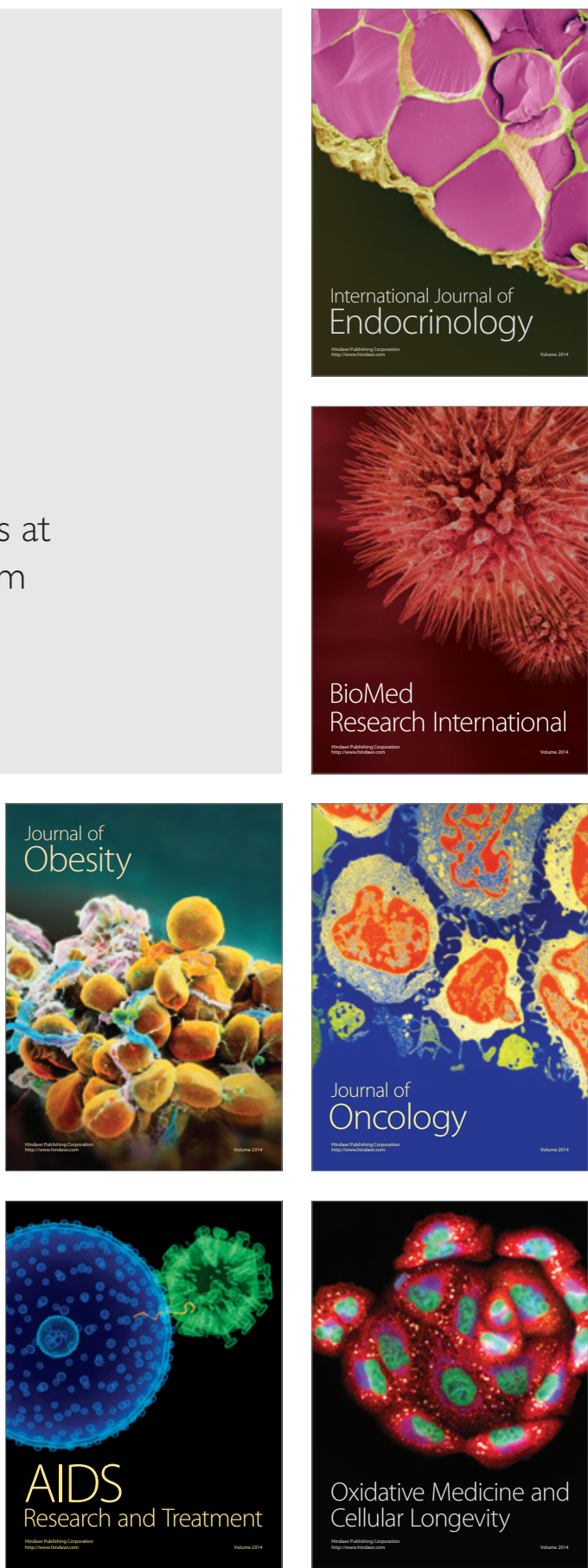